\title{
MaRBLe uncensored: An experience report of the MaRBLe adventure
}

\section{Eline Verrijt-Otten}

\section{Faculty of Psychology and Neuroscience}

Supervisor: Melanie Sauerland

Cohort: 2009

Eline Verrijt-Otten studied psychology at Maastricht University. She took part in the MaRBLe program and conducted a study on eyewitness identification in 2010. She completed her Master's degree in Psychology and Law. In her Master's thesis she examined the association between individual differences in response inhibition ability and false memory proneness. Her main research interests are eyewitness memory, eyewitness identification, reliability of testimony and false memories. Currently she is a job-applicant and enjoying time with her son.

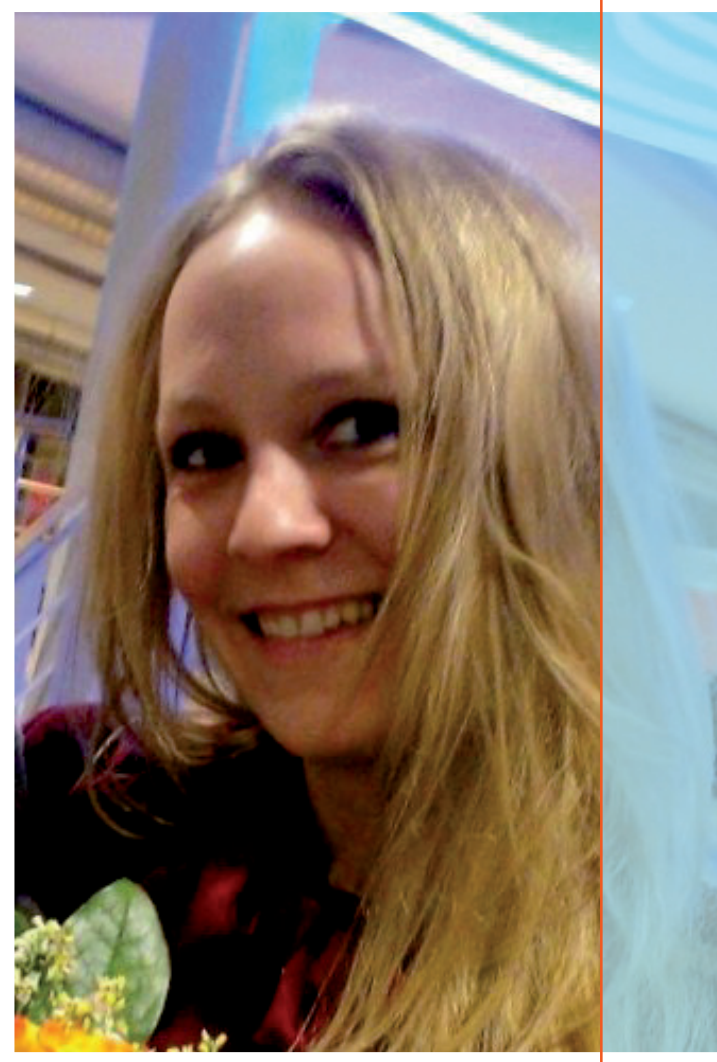


MaRBLe: Maastricht Research-Based Learning, program for excellence. It sounds interesting, intriguing and challenging. Well, it really is all of that and more. Overall it is lots of fun! In the present article I will provide you with an overview of what MaRBLe is. I will describe the different steps of the program, from ups to (occasional) downs. Furthermore, I will briefly explain my MaRBLe study and results.

When I first heard of MaRBLe I did not quite know what it entailed. It did, however, sound tempting enough for me to find out more about it. MaRBLe offers highly motivated and excellent students with research affinity the chance to improve their skills during an intensive research program. In a nutshell, you set up a study under supervision of an expert in the field, exchange and discuss ideas with fellow students, learn in depth about research skills and develop these skills yourself. If you want to apply, there are some requirements you should take into consideration.

First, you need a grade point average of 7.0 and you have to write a letter of motivation. Please do not be discouraged if your GPA is slightly under 7.0. Strong motivation can squeeze you in anyway. I believe motivation and enthusiasm might be equally, if not more important than GPA. However, I am not the one deciding whether you may enrol.

Secondly, you should be interested in research. If you are planning a career in research, or maybe strive for a PhD, MaRBLe would be a great opportunity to develop and elaborate your research skills. Considering my ignorance when it comes to statistics I thought I would never be admitted when I applied. Our MaRBLe coordinator Arie van der Lugt's enthusiasm was contagious, though, and eventually I went home with an overload of motivation and self-esteem. Arie appeared to be an excellent captain of the MaRBLe ship; committed and inspiring with a bunch of humour. Now that I was officially admitted, the next step was to think of a topic and to contact a supervisor.

\section{How to start}

Since I was planning to do the master Psychology and Law, I wanted to do something in the field of eyewitness testimony. I asked Melanie Sauerland to be my supervisor. Her expertise became evident during our first meeting in which she put me in a rollercoaster of information and concepts. We talked about factors that may affect the accuracy of eyewitness descriptions and came up with the idea of examining whether a relationship exists between eating disorders and the accuracy of eyewitness descriptions. Obviously, 
I had some reading to do. I read a pile of articles about perceptual body size distortion, eating disorders and eyewitness testimony as a preparation for the next thing on my to do list: writing a research proposal. In the following I will briefly explain the (initial) rationale of our study.

\section{From perceptual body size distortion to eyewitness description}

Body perception refers to how we perceive our own bodies when looking in the mirror. With perception being a mixture of objective and subjective factors it may deviate between people. However, in some people perception deviates vastly from what most other people perceive. In the 4th ed. of the Diagnostic Statistical Manual of Mental Disorders this phenomenon is called body image disturbance and it is one of the diagnostic criteria for anorexia nervosa and bulimia nervosa (DSM IV, 1994).

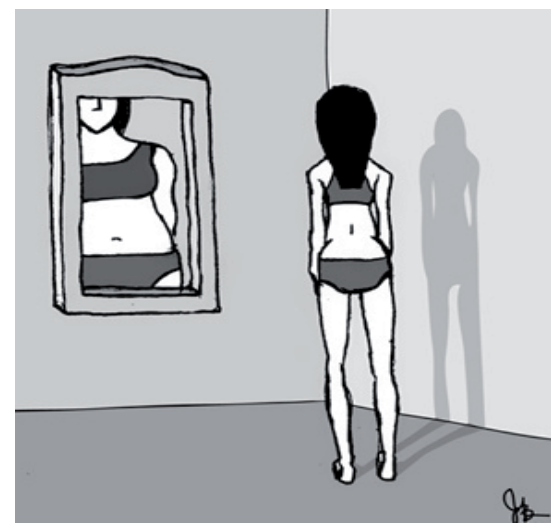

Retrieved from www.tumblr.com/ tagged/ body\%2odistortion

Perceptual body size distortion is present in patients with eating disorders (Schneider, Frieler, Pfeiffer, Lehmkuhl, \& Salbach-Andrae, 2009). Patients generally tend to significantly overestimate their own body size compared to healthy controls (Ferrer-García \& Gutiérrez-Maldonado, 2008). To elaborate on this finding we wondered what would happen if these people were asked to estimate other people's body size? Is body perception distortion merely present in mirror reflections or will difficulties persist? If body image distortion affects perception of other people, it is conceivable that this would affect the capability to make valid person descriptions. Erroneous person descriptions can have an impact on the fairness of lineups constructed on basis of these (erroneous) descriptions. Biased lineups could in turn have an impact on eyewitnesses' identification decisions, convictions and thus jurisdiction.

\section{Writing a research proposal}

Quite interesting, right? I started writing my research proposal but then a substantial hiccup barricaded my way, the first occasional down between all ups. We could not test 
eating disordered patients since this would have to be approved by the medical ethical committee and that would not only cost a lot of time, but also a lot of money. Luckily there was no time to grieve since we had to come up with plan B. We decided to examine whether perceptual body size distortion existed in restrained eaters.

Restrained eaters do not fulfil all requirements to be diagnosed with eating disorder. However, they exhibit extreme dieting behaviour and tend to be preoccupied with their body and weight. A literature search showed that research on this topic was scarce. I did, however, find some evidence of restrained eaters having difficulties in estimating the size of body parts.

A recent study investigated the relationship between perceptual body size estimations and disordered eating symptomatology (Mussap, McCabe, \& Ricciardelli, 2008). It was found that women with elevated levels of restrained eating behaviour were more likely to overestimate their body size. This implies that not only patients with eating disorders, but also individuals at risk of developing an eating disorder or restrained eaters, have perceptual body size distortion. Despite the lack of research there now was reason to believe that eating disordered patients and restrained eaters represent the same population regarding perceptual body size distortion. Subsequently we asked ourselves a crucial question: What happens if eating-disordered patients and restrained eaters are asked to estimate the body size of others? Decades ago, Touyz (1984) examined whether eating disordered patients had difficulties in estimating normal weight models' shape. Results showed that the control group underestimated the models by $5 \%$. Underestimation in the patient group was $20 \%$. According to Touyz, this implies that eating disordered patients do not only misperceive their own bodies, but also display body image distortion regarding other people.

Based on the literature I wrote my research proposal which had to be approved by the ethical committee. Meeting the committee was exciting and somehow scary. I knew beforehand that I would be subjected to critical questions and that made me a little nervous. Not everything was crystal clear to the committee so I tried to explain everything as good as possible. Of course, I survived.

\section{A marble meeting in between}

As our literature search continued and we were waiting for approval, all MaRBLe students gathered for a meeting in order to introduce their research proposals to each other. We shortly presented what we had done so far and exchanged ideas. Meanwhile, everything was filmed by genuine MaRBLe supporter Eri Heesakkers for the next generations of MaRBLe students. It was quite an informal gathering, yet very educational. I personally thought it was a good thing to explain our study in my own words, not only to check whether it was clear for myself but also to gain useful and fresh feedback. A funny detail 
was that we contacted several students who were studying abroad via Skype. If you do not like this kind of meeting you should probably not apply for MaRBLe since your co-students will know where to find you no matter what!

\section{Methodology and procedure}

A nice but demanding part of the MaRBLe project was gathering the experimental supplies and generating measuring tools. We made a movie and lineup pictures and developed several questionnaires as well as a scoring system.

The movie should resemble the witnessing of a crime. I found friends and family willing to be actors for a day. Some of them actually were quite talented. The film shows the theft of a wallet in a bar setting. The four

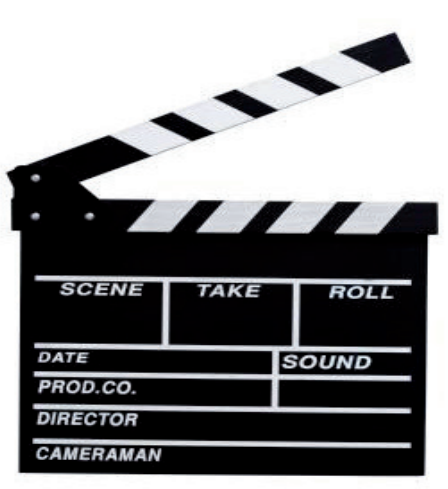
actors varied in outer appearance, age, gender and body size. Shooting the movie was a lot of fun, even after having shot the same scene over and over again.

While editing the movie I received good news: two students working on an individual elective would join and assist me. I thought it was very nice working together. After all, we can learn more from each other than from ourselves. Not only did their help reduce the workload but it was especially nice because we could discuss and exchange ideas. The informal small talk in between was just an extra. Meanwhile, we needed to think of a way to obtain person descriptions of the four actors. We constructed three questionnaires in which the actors were referred to as Bartender (female), Thief (female), Man and Woman. These included a free report in which participants had to describe all actors separately

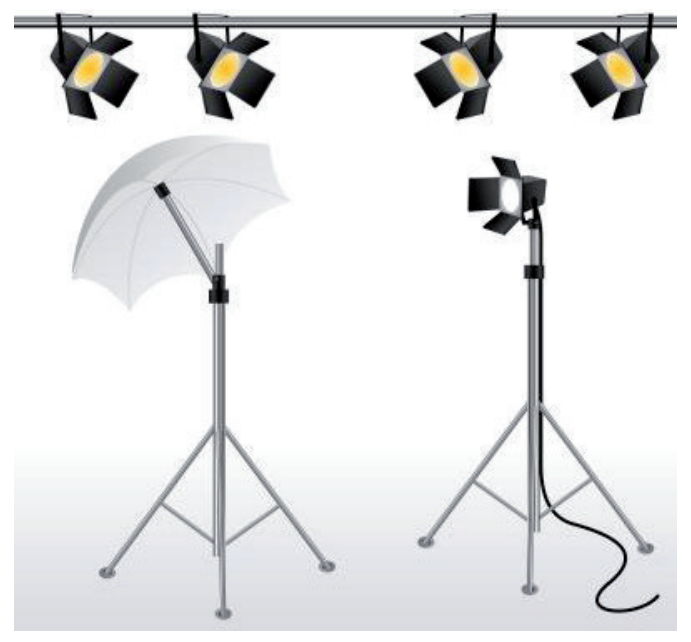
as detailed as possible, additional open questions about the outer appearance of the four actors and multiple choice questions about the appearance of the four actors. We divided the participants into two groups (restrained vs. unrestrained eaters) using the Restrained Scale (Herman, Polivy, Pliner, Threlkeld, \& Munic, 1978). In addition, we developed a 197 variable scoring system in order to code the person descriptions made by participants. 
The next step was to build a photo database. If person descriptions made by restrained eaters would differ significantly from person descriptions of unrestrained eaters we should examine whether lineup fairness differs as a function of restrained eating. In order to create photo lineups we needed pictures. Lots and lots of pictures... For all four of the actors in the movie we needed at least twenty 'lookalikes' who would match the description of the actor. For each person, several pictures were taken (e.g., frontal neutral, frontal smiling, 90 degrees, 45 degrees, frontal full body, frontal full body with hair behind the shoulders) in front of a white background. Taking the pictures was fun to do, but in the end it became more difficult to find suitable matches. Eventually, we did not even use the pictures since no time remained to conduct this part of the study. Surely, not being able to use the pictures was disappointing, but dealing with this kind of misfortune is part of the process as well. Moreover, I know that another student proceeded my work in the photo lab so I am confident that an extensive database will be constructed for future research.

\section{Data collection}

When all materials were prepared, we were finally ready to collect data. Participants were selected based on their scores on the Restrained Scale, as established in the annual first year screening of the Faculty of Psychology and Neuroscience. Students who scored 1.5 standard deviation above $(>15)$ the mean of a Dutch sample on the Restrained Eating Scale were selected as restrained eaters and those who scored 1.5 standard deviation below $(<10)$ were selected as unrestrained eaters. During the experiment, participants watched the film and provided person descriptions using the questionnaires. Testing was a lot of fun, yet problematic at times. It appeared that rooms were sometimes double booked which made us very inventive in improvising testing rooms. I remember even having tested in Melanie's office once. Moreover, there were a couple of participants who did not show up at the arranged time and place.

\section{Data processing}

After having tested the last participant there was still a lot of work waiting for us. With a 197 variable scoring system, scoring the descriptions seemed to take ages. We had two people scoring the same participants in order to establish inter-rater reliability and we put the obtained data in Excel files. Imagine typing an overload of zeros and ones in a file for hours and hours...not the most memorable part of the MaRBLe adventure, especially not when you make a mistake somewhere in between participant number 5 and 27. However, we managed to get the job done decently and were thus ready for the most exciting thing: data analysis! 


\section{Data analysis}

We gathered in Melanie's office for this special moment. Although our study did not confirm our hypothesis, we did however find some interesting trends and results. To mention the most important findings: Restrained eaters were very good in estimating the thin Bartender's weight (in kg), but were unable to assign this weight to the correct label (i.e., skinny, slim, average, chubby, fat). At a BMI of 19, restrained eaters tended to label the Bartender as average built while unrestrained eaters more frequently thought the bartender to be slim. This shows that restrained eaters have difficulties in determining which appearance corresponds to which label.

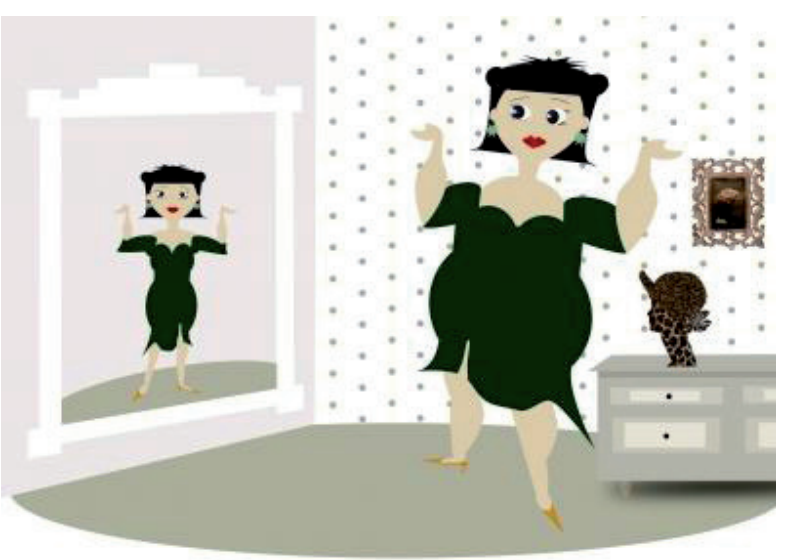

The finding that restrained eaters could estimate the Bartender's weight accurately but did not assign her weight to the most suited descriptor was not found for the other targets. Possibly because she was the only person in the film they could compare themselves with, considering the Man and Woman being older and the Thief having a BMI

of 25.There are several possible explanations for this observation. First, restrained eaters are preoccupied with their own body and weight. They have high body dissatisfaction and want to lose weight because they think they need to be thinner. Restrained eaters may therefore have the misconception that a BMI of 19 is 'average' while it is actually on the lower bound of what is defined as healthy. Another explanation may be that restrained eaters were jealous of the thin Bartender's figure and weight due to their own preoccupation with being thin. As a consequence, they labelled the Bartender's figure as average in order to reduce the discrepancy between the bartender's appearance and their own appearance.

Recently, Glunz (2012) conducted a similar study in which participants (unrestrained vs. intermediate vs. restrained eaters) described four actresses they had previously seen in a movie (different from the one I used) depicting a crime. In addition, they described the crime in general. Results showed no significant difference in eyewitness performance between the three restrained eating groups. There were some interesting findings when looking at details though. Restrained eaters overestimated the weight of the second skinniest actress $(B M I=21.9)$ whereas the other two groups underestimated it. This is in contrast with our results. We found that restrained eaters were more accurate in 
estimating the weight of a thin girl than unrestrained eaters. A possible explanation for the divergent results found in both studies may be that the actors in both movies have differed on dimensions such as sympathy, attractiveness, or distinctiveness. This may have influenced how participants perceived and thus described them. However, future research may provide more insight.

\section{Writing}

With the practical part being finished, I started writing my thesis. I like writing a lot, yet, I found it difficult to stay sharp. My first version came back with an almost frightening amount of notes from my critical (though righteous) supervisor. I dare say her sincere feedback improved my academic writing skills substantially. I enjoyed her as my supervisor since she is very helpful, to the point and always positive. Of course, the fact that she graded me with an 8.0 is pretty nice as well.

\section{Symposium}

Finally then, the icing on the cake: the mini symposium! I made a beautiful poster which I had presented at the Student Research Conference in Leiden earlier. I would say this was the best part of the entire MaRBLe process. We explained our studies and findings to each other and the supervisors during presentations and poster sessions. Very interesting to find out what fellow students experienced and obviously, what their results were. A fine way to finish a great adventure!

Hopefully this report has depicted what MaRBLe entails and maybe it has even incited your enthusiasm. Good luck and enjoy! 


\section{References}

Diagnostic and statistical manual of mental disorders (4th ed.). (1994). Arlington, VA US: American Psychiatric Publishing, Inc.

Ferrer-García, M., \& Gutiérrez-Maldonado, J. (2008). Body image assessment software: Psychometric data. Behavior Research Methods, 40, 394-407.

Glunz, S. (2012). Does restrained eating influence eyewitness performance? Unpublished Master's thesis, Maastricht University, Maastricht, the Netherlands.

Herman, C. P., Polivy, J., Pliner, P., Threlkeld, J., \& Munic, D. (1978). Distractibility in dieters and nondieters: An alternative view of 'externality'. Journal of Personality and Social Psychology, 36, 536-548.

Mussap, A. J., McCabe, M. P., \& Ricciardelli, L. A. (2008). Implications of accuracy, sensitivity, and variability of body size estimations to disordered eating. Body Image, 5, 80-90.

Otten, E. (2010). Does restrained eating affect eyewitnesses' description? Unpublished Bachelor's thesis, Maastricht University, Maastricht, the Netherlands.

Schneider, N., Frieler, K., Pfeiffer, E., Lehmkuhl, U., \& Salbach-Andrae, H. (2009). Comparison of body size estimation in adolescents with different types of eating disorders. European Eating Disorders Review, 17, 468-475.

Touyz, S. W. (1984). Body shape perception and its disturbance in anorexia nervosa. British Journal of Psychiatry, 144, 167-171. 